\title{
Rhinal Cortices Connecting the Papez and Yakovlev Circuits: An Integrated Viewpoint Through MRI Epidemiology to Animal Experiment for Better Understanding Clinical Features of Alzheimer Disease and Vascular Dementia
}

\author{
Kenichi Meguro* \\ Geriatric Behavioral Neurology, Tohoku University New Industry Creation Hatchery Center, Tohoku University, Tohoku University Graduate \\ School of Medicine, Japan \\ *Corresponding author: Kenichi Meguro, Project Leader and Professor, Geriatric Behavioral Neurology Project, Tohoku University \\ New Industry Creation Hatchery Center, Cyclotron Radioisotope Center, Tohoku University, Tohoku University Graduate School of \\ Medicine, Seiyo-machi, IDAC, Aoba-ku, 980-8575 Sendai, Japan
}

\section{Introduction}

I herein present a unique statement, not focusing on the specific area but present an integrated viewpoint through MRI epidemiology to animal experiment. The aim is to provide a perspective for better understanding clinical features of Alzheimer disease (AD) and vascular dementia (VaD). It is well known that $\mathrm{AD}$ and $\mathrm{VaD}$ are two major dementing diseases [1,2], and the clinical features are different; grossly, AD shows disintegrated emotion and cognitive function, and VaD shows apathy. First, I will present two typical VaD cases.

\section{Case Reports of VaD with Thalamic Infarctions}

Case-1: A 71-year-old right-handed woman [3]

She was originally scrupulous in her performance of domestic chores. She suddenly developed difficulty finding words and used many pronouns in her communication. She exhibited apathy and did her housework in a careless manner. Although her MMSE score was 23, she could not recall episodic memory of her daily life. Neurologically, she exhibited very slight right hemiparesis. MRI showed infarction in the left thalamic anteroventral nucleus (AN), and SPECT revealed decreased bilateral frontal and anterior cingulate blood flow. The precuneus and parietotemporal areas, characteristic of $\mathrm{AD}$, were intact. Thus, the diagnosis is $\mathrm{VaD}$, and her amnesia was explained by the disturbed Papez circuit involving AN as illustrated in the right part of (Figure 1).

\section{Case-2: An 82-Year-Old Right-Handed Man [3]}

He had been active and enjoyed his hobbies every day. He suddenly developed difficulty finding words, gradually exhibited amnesia and apathy. His MMSE score was 22. He could poorly remember episodic memory of his daily life. Neurologically, he exhibited slight right hemiparesis. MRI showed infarction in the left thalamic dorsomedial nucleus (MD), and SPECT revealed decreased bilateral frontal and anterior cingulate blood flow. The precuneus and parietotemporal areas were intact. Thus the diagnosis is VaD; however, one of the fronto-subcortical circuits involving MD cannot explain amnesia, since clinically we know that the limited frontal lesions cannot manifest amnesia. The MD is involved in the Yakovlev circuit responsible for emotion. Then how we can consider his amnesia? To get straight to the point, preserved rhinal cortices can connect the Papez and Yakovlev circuits, and explain amnesia as a remote effect as illustrated in (Figure 1). Then I will present MRI epidemiology on the entorhinal cortex.

MRI Epidemiology Disclosed an Importance of

\section{Entorhinal Cortex}

To investigate MRI findings of questionable dementia, 485 participants were selected in a community including 113 Clinical Dementia Rating (CDR) 0.5 people in my early study [4]. We found that each part of the brain showed atrophy compared with healthy 
adults. An amygdala atrophy was the only finding indicating CDR effect but no age effect. The amygdala or anterior entorhinal atrophy was found to be important for discriminating questionable dementia from healthy elderly. Using more sophisticated methodology [5], we computed cerebral cortical thickness, correlating with memory performance in questionable dementia people. We found that poor episodic memory was associated with thinner cortices in the left entorhinal region. Then the entorhinal cortex can affect remote cortical areas through neuronal network?

\section{Functional Neuroimaging and an Animal Experiment}

Hippocampal area (including entorhinal cortex) atrophy and hypometabolism in the posterior association neocortex are two well-known neuroimaging features of AD. The latter was already revealed in the CDR 0.5 stage further decline to AD [6]. Analyzing the data of MRI based atrophy and PET based glucose metabolism, we found a positive neurobiologically expected correlation between hippocampal width and angular gyrus metabolism.
Hippocampal-neocortical disconnection due to medial temporal pathology may at least partly explain the posterior association cortex hypometabolism found in $\mathrm{AD}[7,8]$.

To exclude an indirect dementia severity effect and directly prove the effect of neuronal network, we performed an animal experiment $[9,10]$. Namely, the remote metabolic effects of bilateral neurotoxic lesions of rhinal cortices was assessed. Using PET, glucose metabolism was measured before surgery and a couple months afterward. Compared with sham-operated baboons, the lesioned animals showed a long-lasting metabolic decline in the parietal and temporal cortices, and posterior hippocampal region, all specific to $\mathrm{AD}$. This shows the temporoparietal and hippocampal hypometabolism found in AD may partly result from neuroanatomical disconnection with the rhinal cortex. Neuronal damage and dysfunction in the rhinal cortices is suggested to play a major role in the expression of AD. Then finally, the concept for better understanding clinical features of $\mathrm{AD}$ and vascular $\mathrm{VaD}$ is summarized in (Figure 1).

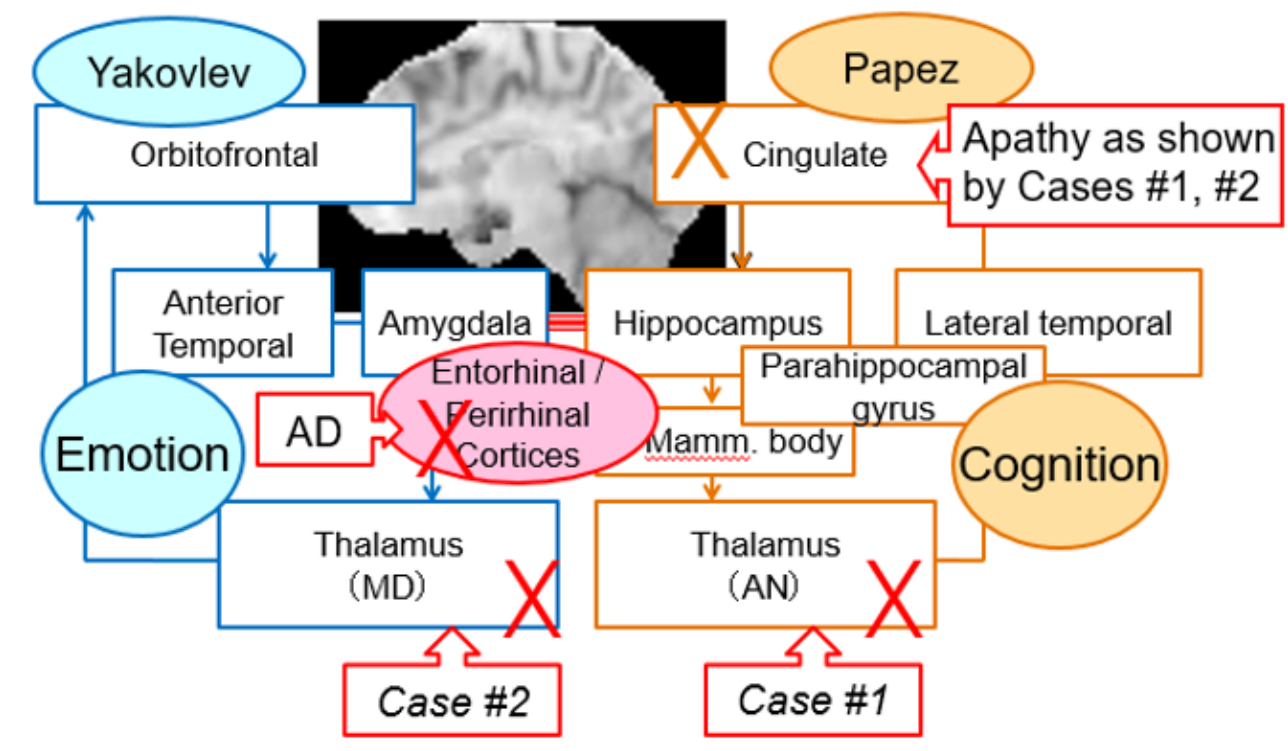

Figure 1.

\section{Functional Neuronal Networks for Better Understanding of $\mathrm{AD}$ and $\mathrm{VaD}$}

Clinically $\mathrm{AD}$ patients frequently shows behavioral and psychological symptoms of dementia (BPSD), despite relatively preserved cognitive function in the mild stage. This imbalance between emotion and cognition may be based on the disconnection between the Yakovlev and Papez circuits, which are responsible for emotional and cognitive functions, respectively.
On the contrary, VaD patients show mainly apathy and less frequently such unbalanced, disintegrated emotional changes disproportionally to cognitive level unlikely to $\mathrm{AD}$ patients. This is probably due to spared rhinal cortices.

\section{References}

1. Meguro K, Ishii H, Yamaguchi S, Ishizaki J, Shimada M, et al. (2002) Prevalence of dementia and dementing diseases in Japan: The Tajiri Project. Archives of Neurology 59(7): 1109-1114. 
2. Meguro K, Tanaka N, Kasai M, Nakamura K, Ishikawa H, et al. (2012) Prevalence of dementia and dementing diseases in the old-old population in Japan: The Kurihara Project. Implications for Long-Term Care Insurance Data. Psychogeriatrics 12(4): 226-234.

3. Meguro K, Akanuma K, Ouchi Y, Meguro M, Nakamura K, et al. (2013) Vascular dementia with left thalamic infarction: Neuropsychological and behavioral implications suggested by involvement of the thalamic nucleus and the remote effect on cerebral cortex. The Osaki-Tajiri Project. Psychiatry Research: Neuroimaging 213(1): 56-62.

4. Ishii H, Meguro K, Yamaguchi S, Hirayama K, Tabuchi M, et al. (2006) Different MRI findings for normal elderly and very mild Alzheimer's disease in a community: Implications for clinical practice. The Tajiri Project. Archives of Gerontology and Geriatrics 42(1): 59-71.

5. Fujishima M, Maikusa N, Nakamura K, Nakatsuka M, Matsuda $H$, et al. (2014) Mild cognitive impairment, poor episodic memory, and late-life depression are associated with cerebral cortical thinning and increased white matter hyperintensities. Frontiers in Aging Neuroscience 6: 306.

6. Ishii H, Ishikawa H, Tashiro M, Yamaguchi S, Meguro K (2009) Decreased cortical glucose metabolism in converters from CDR 0.5 to Alzheimer's disease in a community: The Osaki-Tajiri Project. International Psychogeriatrics 21(1): 148-156.
7. Yamaguchi S, Meguro K, Itoh M, Hayasaka C, Shimada M, et al. (1997) Decreased cortical glucose metabolism correlated with hippocampal atrophy in Alzheimer's disease as shown by MRI and PET. Journal of Neurology Neurosurgery and Psychiatry 62(6): 596-600.

8. Meguro K, LeMestric C, Landeau B, Desgranges B, Eustache F, et al (2001) Relations between hypometabolism in the posterior association neocortex and hippocampal atrophy in Alzheimer's disease: A PET/MRI correlative study. Journal of Neurology Neurosurgery and Psychiatry 71(3): 315-321.

9. Meguro K, Blaizot X, Kondoh Y, LeMestric C, Baron JC, et al. (1999) Neocortical and hippocampal glucose hypometabolism following neurotoxic lesions of the entorhinal and perirhinal cortices in the nonhuman primate as shown by PET: Implications for Alzheimer's disease. Brain 122(Pt 8): 1519-1531.

10. Blaizot X, Meguro K, Millien I, Baron JC, Chavoix C (2002) Correlations between visual recognition memory and neocortical and hippocampal glucose metabolism after bilateral rhinal cortex lesions in the baboon: Implications for Alzheimer's disease. J Neurosci 22(21): 9166-9170.

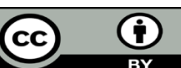

This work is licensed under Creative Commons Attribution 4.0 License

To Submit Your Article Click Here: Submit Article
DOI: $10.32474 /$ JJNBD.2020.04.000193

Online Journal of Neurology
and Brain Disorders
Assets of Publishing with us

